\title{
Sources of variance of downwelling irradiance in water
}

\author{
Peter Gege ${ }^{1, *}$ and Nicole Pinnel ${ }^{2}$ \\ ${ }^{1}$ German Aerospace Center (DLR), Remote Sensing Technology Institute, Oberpfaffenhofen, D-82234 Wessling, Germany \\ ${ }^{2}$ German Aerospace Center (DLR), German Remote Sensing Data Center, Oberpfaffenhofen, D-82234 Wessling, Germany \\ ${ }^{*}$ Corresponding author: Peter.Gege@dlr.de
}

Received 7 December 2010; revised 5 February 2011; accepted 7 February 2011; posted 8 February 2011 (Doc. ID 139147); published 16 May 2011

\begin{abstract}
The downwelling irradiance in water is highly variable due to the focusing and defocusing of sunlight and skylight by the wave-modulated water surface. While the time scales and intensity variations caused by wave focusing are well studied, little is known about the induced spectral variability. Also, the impact of variations of sensor depth and inclination during the measurement on spectral irradiance has not been studied much. We have developed a model that relates the variance of spectral irradiance to the relevant parameters of the environmental and experimental conditions. A dataset from three German lakes was used to validate the model and to study the importance of each effect as a function of depth for the range of 0 to $5 \mathrm{~m}$. (C) 2011 Optical Society of America

OCIS codes: $\quad 010.4450,030.6600,120.6200,280.4788,300.0300$.
\end{abstract}

\section{Introduction}

The determination of optical properties of water bodies under natural illumination conditions requires a measurement of the downwelling spectral irradiance, $E_{d}(\lambda)$. However, if this measurement is performed below the water surface, it can introduce a large error, because $E_{d}$ usually shows very high short-term variability due to focusing and defocusing of sunlight by surface waves [1,2]. These waveinduced irradiance variations depend strongly on wind speed, solar elevation, and depth [3-5] and are most pronounced at a relatively smooth water surface and clear sky $[6,7]$. Under these conditions, variability is typically in the order of $20 \%$ to $40 \%$ near the water surface $[4,8]$, but flashes with durations of ten to several tens of milliseconds can increase the intensity up to a factor of 5 [4]. The strongest fluctuations were observed in the upper $2 \mathrm{~m}$ [8].

It can be an advantage to avoid the wave focusing effect by measuring $E_{d}(\lambda)$ above the water surface, e.g., for the determination of subsurface reflectance $[9,10]$. But for some optical properties such as the diffuse attenuation coefficient or bottom reflectance,

0003-6935/11/152192-12\$15.00/0

(C) 2011 Optical Society of America in-water measurements are unavoidable. In these cases, the large variability of irradiance can introduce significant errors to the derived parameters. It is very difficult to quantify the error, and in particular its wavelength dependency, because, to our knowledge no model exists so far that relates the variability of the $E_{d}$ intensity and spectral shape to parameters of the wave focusing effect that can be determined easily during a field campaign. We have developed such a model and applied it to a large dataset from three German lakes to study the variability of intensity and spectral shape of irradiance in the depth range from 0 to $5 \mathrm{~m}$ and to quantify the impact of environmental (wave focusing, wave-induced changes in the water column's height above the sensor) and experimental conditions (tilted irradiance sensor, variable sensor orientation).

\section{Model}

\section{A. Parameterization of Irradiance}

The downwelling irradiance for an observer in air or in water $\left(E_{d}\right)$ is the sum of a direct $\left(E_{d d}\right)$ and a diffuse $\left(E_{d s}\right)$ component, where the direct component represents that part of the extraterrestrial solar irradiance that has been directly transmitted through the atmosphere and eventually the water along 
the line of sight between the Sun disk and observer, and the diffuse component is the radiation that reaches the observer from the other directions due to scattering in the atmosphere. We express this sum as follows:

$$
E_{d}(\lambda, z)=f_{d d} E_{d d}(\lambda, z)+f_{d s} E_{d s}(\lambda, z),
$$

where $\lambda$ denotes wavelength and $z$ is the sensor depth. The $\lambda$ symbol is omitted in most equations for brevity. The parameters $f_{d d}$ and $f_{d s}$ describe the intensity changes of $E_{d d}$ and $E_{d s}$ compared to conditions with undisturbed illumination geometry. For an observer in air, these reference conditions (with $f_{d d}=f_{d s}=1$ ) are defined by a cloudless atmosphere and unobscured sky view, for an observer in water additionally by a plane water surface without wave focusing. $0 \leq f_{d d}<1$ corresponds to measurements when waves or obstacles decrease the magnitude of the direct component (shadowing effect), $f_{d d}>1$ when $E_{d d}$ intensity is increased (wave focusing effect). Likewise, a decrease of the diffuse component compared to undisturbed geometry is described by $0 \leq f_{d s}<1$, and an increase by $f_{d s}>1$.

The diffuse component at depth $z$ is related to that below the surface as follows:

$$
E_{d s}(z)=E_{d s}(0-) \exp \left\{-K_{d s} z l_{d s}\right\} .
$$

The symbol 0 - indicates that the sensor is in water and just beneath the water surface. $K_{d s}$ is the average diffuse attenuation coefficient of the water column between the depths $0-$ and $z$. A factor $l_{d s}$ is introduced as the average path length of diffuse radiation.

The direct component is attenuated along a path with length $z / \cos \theta_{\text {Sun }}$ :

$$
E_{d d}(z)=E_{d d}(0-) \exp \left\{-\frac{K_{d d} z}{\cos \theta_{\text {Sun }}^{\prime}}\right\} .
$$

$K_{d d}$ is the average attenuation coefficient for direct radiation between the depths $0-$ and $z$. The Sun zenith angle in water, $\theta_{\text {Sun }}^{\prime}$, is related to that in air, $\theta_{\text {Sun }}$, by Snell's law $n_{w} \sin \theta_{\text {Sun }}^{\prime}=\sin \theta_{\text {Sun }}$, with $n_{w}$ denoting the refractive index of water.

\section{B. Variance of Irradiance}

The change of $E_{d}$ at depth $z$ during a measurement is expressed by the differential $d E_{d}$ of Eq. (1):

$$
\begin{aligned}
d E_{d}(z)= & d f_{d d} E_{d d}(z)+f_{d d} d E_{d d}(z)+d f_{d s} E_{d s}(z) \\
& +f_{d s} d E_{d s}(z) .
\end{aligned}
$$

The four terms of the equation describe the four sources of variability: changes of the direct $\left(d E_{d d}\right)$ and diffuse $\left(d E_{d s}\right)$ irradiance components caused by intensity changes just below the surface or by variations of sensor depth, and variable weighting factors $\left(d f_{d d}, d f_{d s}\right)$ provoked by waves.

The change of $E_{d d}$ during a measurement is obtained from the differential of Eq. (3):

$$
\begin{aligned}
d E_{d d}(z)= & {\left[d E_{d d}(0-)-E_{d d}(0-) \frac{K_{d d}}{\cos \theta_{\text {Sun }}^{\prime}} d z\right] } \\
& \times \exp \left\{-\frac{K_{d d} z}{\cos \theta_{\text {Sun }}^{\prime}}\right\} .
\end{aligned}
$$

$K_{d d}$ and $\theta^{\prime}$ Sun are assumed constant during the measurement, i.e., $d K_{d d}=0$ and $d \theta_{\text {Sun }}^{\prime}=0$ was set. $E_{d d}(0-)$ is proportional to the cosine of the incidence angle of the Sun's rays on the detector:

$$
E_{d d}(0-)=E_{d d}^{0} \cos \left(\theta^{\prime}{ }_{\text {Sun }}+\theta_{s}\right),
$$

where $E_{d d}^{0}$ denotes the irradiance for $\theta_{\text {Sun }}^{\prime}=0$ and $\theta_{s}=0$. This equation accounts for a potential tilt of the $E_{d}$ sensor by an angle $\theta_{s}$ relative to a horizontal plane. It follows

$$
\begin{aligned}
d E_{d d}(0-) & =-E_{d d}^{0} \sin \left(\theta^{\prime}{ }_{\text {Sun }}+\theta_{s}\right) d \theta_{s} \\
& =-E_{d d}(0-) \tan \left(\theta_{\text {Sun }}^{\prime}+\theta_{s}\right) d \theta_{s},
\end{aligned}
$$

and further

$d E_{d d}(z)=-E_{d d}(z)\left[\tan \left(\theta_{\text {Sun }}^{\prime}+\theta_{s}\right) d \theta_{s}+\frac{K_{d d}}{\cos \theta_{\text {Sun }}^{\prime}} d z\right]$.

For the diffuse irradiance, it is assumed that neither waves nor a tilted sensor change the average incidence angle on the detector significantly; i.e., $E_{d s}(0-)$ is assumed constant. This assumption implies that the changes of the angular radiance distribution induced by waves and sensor tilt are small. Using $d E_{d s}(0-)=0$ and $d l_{d s}=0$, it follows from Eq. (2):

$$
d E_{d s}(z)=-K_{d s} E_{d s}(z) l_{d s} d z \text {. }
$$

By introducing the ratio of direct to diffuse irradiance,

$$
r_{d}(z)=\frac{f_{d d} E_{d d}(z)}{f_{d s} E_{d s}(z)}
$$

$E_{d d}$ and $E_{d s}$ can be expressed in terms of $E_{d}$ as follows:

$$
\begin{aligned}
& E_{d d}(z)=\frac{r_{d}(z)}{r_{d}(z)+1} \frac{E_{d}(z)}{f_{d d}}, \\
& E_{d s}(z)=\frac{1}{r_{d}(z)+1} \frac{E_{d}(z)}{f_{d s}} .
\end{aligned}
$$

Combining the above equations leads to

$$
\begin{aligned}
d E_{d}(z) & =-E_{d}(z) \frac{r_{d}(z)}{r_{d}(z)+1}\left[\tan \left(\theta_{\text {Sun }}^{\prime}+\theta_{s}\right) d \theta_{s}\right. \\
& \left.+\frac{K_{d d}}{\cos \theta_{\text {Sun }}^{\prime}} d z-\frac{d f_{d d}}{f_{d d}}\right]-\frac{E_{d}(z)}{r_{d}(z)+1}\left[K_{d s} l_{d s} d z-\frac{d f_{d s}}{f_{d s}}\right] .
\end{aligned}
$$


Rearranging gives

$$
\begin{aligned}
\frac{d E_{d}(z)}{E_{d}(z)}= & \frac{r_{d}(z)}{r_{d}(z)+1} \frac{d f_{d d}}{f_{d d}}-\frac{r_{d}(z)}{r_{d}(z)+1} \tan \left(\theta_{\text {Sun }}^{\prime}+\theta_{s}\right) d \theta_{s} \\
& -\frac{1}{r_{d}(z)+1}\left[\frac{K_{d d} r_{d}(z)}{\cos \theta_{\text {Sun }}^{\prime}}+l_{d s} K_{d s}\right] d z \\
& +\frac{1}{r_{d}(z)+1} \frac{d f_{d s}}{f_{d s}} .
\end{aligned}
$$

This equation parameterizes the relative change of $E_{d}$ as a function of variable wave focusing $\left(d f_{d d}\right.$, $\left.d f_{d s}\right)$, changing sensor orientation $\left(d \theta_{s}\right)$ and variable sensor depth $(d z)$ during the measurement. Expressing the equation as a variance leads to the following result: tively; $\rho_{d d}$ and $\rho_{d s}$ are the reflectance factors of direct and diffuse radiation at the water surface, respectively; and $F_{a}$ is the aerosol forward scattering probability. More details are given in [11], where the equations for $T_{r}, T_{a s}$, and $F_{a}$ are taken from [12].

Equation (12) shows that the wavelength dependency of $r_{d}(0-)$ is determined by the scattering components of the atmosphere but not by its absorbing components. Consequently, the distinctive spectral gradients of $E_{d}$, originating from the extraterrestrial solar irradiance and the absorbing components of the atmosphere, are not present in $r_{d}(0-)$. Rather, $r_{d}(0-)$ has a smooth spectral shape, as illustrated in Fig. 3. For depth $z$, the following relationship is obtained [11]:

$$
\begin{aligned}
\operatorname{var}\left[\frac{\Delta E_{d}(\lambda, z)}{E_{d}(\lambda, z)}\right]= & {\left[\frac{r_{d}(\lambda, z)}{r_{d}(\lambda, z)+1}\right]^{2} \operatorname{var}\left[\frac{\Delta f_{d d}}{f_{d d}}\right]+\left[\frac{r_{d}(\lambda, z)}{r_{d}(\lambda, z)+1}\right]^{2} \tan ^{2}\left(\theta_{\text {Sun }}^{\prime}+\theta_{s}\right) \operatorname{var}\left[\theta_{s}\right] } \\
& +\left[\frac{1}{r_{d}(\lambda, z)+1}\right]^{2}\left[\frac{K_{d d}(\lambda) r_{d}(\lambda, z)}{\cos \theta^{\prime}{ }_{\text {Sun }}}+l_{d s} K_{d s}(\lambda)\right]^{2} \operatorname{var}[z]+\left[\frac{1}{r_{d}(\lambda, z)+1}\right]^{2} \operatorname{var}\left[\frac{\Delta f_{d s}}{f_{d s}}\right] .
\end{aligned}
$$

The equation shows that a variable direct component of irradiance $\left(\operatorname{var}\left[\Delta f_{d d} / f_{d d}\right] \neq 0\right)$, caused for instance by the wave focusing effect, induces a spectral change to downwelling irradiance that is characterized by the ratio $\left[r_{d}(\lambda) /\left(r_{d}(\lambda)+1\right)\right]^{2}$. Measurement errors caused by a tilted $\left(\theta_{s} \neq 0\right)$ or a swaying $\left(\operatorname{var}\left[\theta_{s}\right] \neq 0\right) E_{d}$ sensor have the same spectral characteristics; hence, analysis of $E_{d}$ measurements cannot distinguish between these effects. Because the wave focusing effect alters the illumination geometry and a tilted or swaying sensor the viewing geometry, the ratio $\left[r_{d} /\left(r_{d}+1\right)\right]^{2}$ is called henceforth the geometry factor.

If the diffuse component of irradiance is changing $\left(\operatorname{var}\left[\Delta f_{d s} / f_{d s}\right] \neq 0\right)$, the irradiance variance is proportional to $1 /\left(r_{d}(\lambda)+1\right)^{2}$. A variable sensor depth $(\operatorname{var}[z] \neq 0)$ induces the most complex changes to the irradiance spectrum, with dependencies on $r_{d}(\lambda)$, $\theta^{\prime}{ }_{\text {Sun }}, K_{d d}(\lambda)$, and $K_{d s}(\lambda)$. The wavelength dependency and typical magnitudes of the factors of $\operatorname{var}\left[\Delta E_{d} / E_{d}\right]$ are illustrated below in Section $\underline{4}$ (Fig. $\underline{2}$ ).

\section{Ratio of Direct to Diffuse Irradiance}

According to Eq. (11), the ratio of direct to diffuse irradiance, $r_{d}$, is a key parameter of irradiance variance. Just below the water surface, this ratio is given by [11]

$$
r_{d}(0-)=\frac{f_{d d}}{f_{d s}} \frac{2 T_{r} T_{a s}\left(1-\rho_{d d}\right)}{\left[1-T_{r}^{0.95}+2 T_{r}^{1.5}\left(1-T_{a s}\right) F_{a}\right]\left(1-\rho_{d s}\right)} .
$$

$T_{r}$ and $T_{a s}$ are the transmittance spectra of the atmosphere after Rayleigh and aerosol scattering, respec-

$$
r_{d}(z)=r_{d}(0-) \exp \left\{\left(l_{d s} K_{d s}-\frac{K_{d d}}{\cos \theta_{\text {Sun }}^{\prime}}\right) z\right\} .
$$

The wavelength dependency of $r_{d}(0-)$ is altered at $\operatorname{depth} z>0$ by a factor whose spectral shape is determined by $K_{d s}$ and $K_{d d}$. This depth dependency of $r_{d}$ is illustrated in Fig. 3.

\section{Attenuation}

A beam of light passing a water layer is affected by absorption and scattering processes along its path, resulting in spectrally dependent intensity changes. For irradiances, a diffuse attenuation coefficient $K$ parameterizes the average changes along the various paths. The bulk coefficient for $E_{d}$, denoted $K_{d}$, has been studied extensively (see [13] for an overview), but we are not aware of analytic models for the coefficients $K_{d s}$ and $K_{d d}$ as defined by Eqs. (2) and (3), respectively.

Because an irradiance sensor detects radiation from angles covering a hemisphere, only a part of the photons that are scattered out of the incident direction is lost for detection. For a beam incident perpendicular on an irradiance sensor, these are the backscattered photons. They are parameterized by the backscattering coefficient $b_{b}(\lambda)$, which measures the resulting decrease of photon flux per length (in units of $\mathrm{m}^{-1}$ ). Hence, the following approximation is made:

$$
K_{d s}(\lambda)=K_{d d}(\lambda)=a(\lambda)+b_{b}(\lambda),
$$

with $a(\lambda)$ denoting the absorption coefficient of the water layer. Equation (14) corresponds to a widely 
used approximation of the wavelength dependency of $K_{d}(\lambda)$ [14]. However, the $b_{b}$ term is exactly valid only for the idealized condition of perpendicular incidence of all radiation, which is never the case during in situ measurements. For beams with non-nadir incidence, a portion of backscattered photons is detected, and a fraction of the forward scattered radiation gets undetectable. In order to validate the approach, radiative transfer simulations using the well-established model Hydrolight [15] were performed for different depths and Sun zenith angles [11]. These confirm that Eq. (14) describes the wavelength dependency of $K_{d s}$ and $K_{d d}$ with high accuracy.

Hydrolight simulations were further made to determine the average path length for diffuse radiation. The result was $l_{d s}=1.18 \pm 0.05$ for $z$ in the range 0.5 to $5 \mathrm{~m}$ and $\theta_{\text {Sun }}$ between $20^{\circ}$ and $60^{\circ}$ [11].

The optical properties of water are calculated as follows (for $\lambda$ in units of nanometers):

$$
\begin{aligned}
a(\lambda) & =a_{W}(\lambda)+C a_{p h} *(\lambda)+Y \exp [-S(\lambda-440)], \\
b_{b}(\lambda) & =b_{b, W}(\lambda)+X b_{b, X} * .
\end{aligned}
$$

$a_{W}(\lambda)$ and $b_{b, W}(\lambda)$ are the absorption and backscattering coefficients of pure water, respectively. The spectrum $a_{W}(\lambda)$ used here is a combination from different sources: $350-390 \mathrm{~nm}$, interpolation between Quickenden and Irvin [16] and Buiteveld et al. [17]; 391-787 nm, Buiteveld et al. [17]; 788-874 nm, our own unpublished measurements on UV-treated pure water; 875-1000 nm, Palmer and Williams [18]. For $b_{b, W}(\lambda)$, the relation of Morel [19] is used: $\overline{b_{b, W}}(\lambda)=$ $b_{1}(\lambda / 500)^{-4.32}$ ( $\lambda$ in $\left.\mathrm{nm}\right)$ with $\overline{b_{1}}=0.00111 \mathrm{~m}^{-1}$ for fresh water.

Three types of water constituents are considered: phytoplankton, gelbstoff (also known as yellow substance or colored dissolved organic matter), and suspended particles. Phytoplankton and gelbstoff are treated as pure absorbers, and suspended particles are treated as pure scatterers. Phytoplankton concentration, $C$, is expressed as mass of the pigments chlorophyll-a plus pheophytin-a per water volume $\left(\mathrm{mgm}^{-3}\right)$, its specific absorption coefficient, $a_{p h} *(\lambda)$, is species dependent. Here, a spectrum $a_{p h} *(\lambda)$ is used that represents a typical phytoplankton mixture for Lake Constance [20,21]. Gelbstoff concentration, $Y$, is expressed in units of absorption at $440 \mathrm{~nm}\left(\mathrm{~m}^{-1}\right)$; its specific absorption coefficient is approximated by an exponential function with spectral slope $S=0.014 \mathrm{~nm}^{-1}$, which is representative of a great variety of water types $[\underline{22}, \underline{23}]$. The concentration of suspended particles, $X$, is expressed as mass per water volume $\left(\mathrm{gm}^{-3}\right)$. Backscattering of suspended particles is approximated as a wavelengthindependent function with $b_{b, X^{*}}=0.0086 \mathrm{~m}^{2} \mathrm{~g}^{-1}$, which is representative for Lake Constance [21].

\section{Materials and Methods}

\section{A. Instrumentation}

A commercial irradiance sensor manufactured by TriOS (Oldenburg, Germany) was used for the measurements. It is part of a sensor system that consists of three submersible hyperspectral radiometers to obtain simultaneous underwater measurements of downwelling irradiance $\left(E_{d}\right)$, upwelling irradiance, and upwelling radiance. Only the $E_{d}$ data are used in this paper. The $E_{d}$ sensor, which is from the series RAMSES-ACC-VIS (serial number SAM_8109; SAM 806 F on 26 June 2004), measures the downwelling spectral irradiance from 320 to $950 \mathrm{~nm}$ at a spectral sampling interval of $3.3 \mathrm{~nm}$ with a $7 \mathrm{~mm}$ diameter cosine collector.

A two-axis inclination module with pressure add-on (TriOS, serial number ADM_C030) is mounted inside the $E_{d}$ sensor housing. It provides simultaneously to each data take information about sensor depth (at 0.1 bar resolution, corresponding to $10 \mathrm{~cm}$ water column) and inclination (at $1^{\circ}$ resolution). Because the axes were not aligned relative to the rod axis, only total inclination is used.

\section{B. Dataset}

A dataset of 421 downwelling irradiance measurements was collected in 2003 and 2004 at the German lakes Bodensee (Lake Constance), Starnberger See, and Waginger See. Each measurement consists of 4 to 50 individual data takes at a similar sensor depth. The number of all single spectra is 4375 ; the average per measurement is 11 . The measurement time varied from 21 to $700 \mathrm{~s}$ with an average of $105 \mathrm{~s}$. Integration time and repetition rate were set automatically by the instrument electronics. Realized values of the integration time were $8,16,32,64$, 128,256 , and $512 \mathrm{~ms} .98 \%$ of the data has integration times between 16 and $64 \mathrm{~ms}(16 \mathrm{~ms}, 38 \%$ of the data; $32 \mathrm{~ms}, 45 \%$; $64 \mathrm{~ms}, 15 \%$ ); the average is $34 \mathrm{~ms}$.

The campaigns were performed mostly in shallow waters using a small boat that was slightly swaying during data acquisition due to wind and waves. The Sun zenith angle ranged from $24^{\circ}$ to $66^{\circ}$; the average was $38.5^{\circ}$. The sensor system was placed at a distance of 2 to $3 \mathrm{~m}$ from the boat, beyond its shading influence. Measurements were performed in various depths ranging from 0 to $5 \mathrm{~m}$. A $25 \mathrm{~m}$ long cable was used to transfer the data to an on-board computer for storage. Figure 1 provides a summary of the measurement conditions as derived from the pressure and inclination sensors.

The averages are $0.67 \mathrm{~m}$ for sensor depth and $5.8^{\circ}$ for inclination. The respective standard deviations during a measurement are $0.043 \mathrm{~m}$ and $1.6^{\circ}$, corresponding to variances of $0.0019 \mathrm{~m}^{2}$ and $0.00074 \mathrm{sr}^{2}$. Hence, $z=0.7 \mathrm{~m}, \theta_{\text {Sun }}=40^{\circ}$ and $\operatorname{var}\left[\theta_{s}\right]=0.0007 \mathrm{sr}^{2}$ are used in the simulations in Section 4 to represent typical measurement conditions (see Table 1 ). Because the relative $z$ values can be determine $\bar{d}$ more accurately from the $E_{d}$ measurements by inverse 

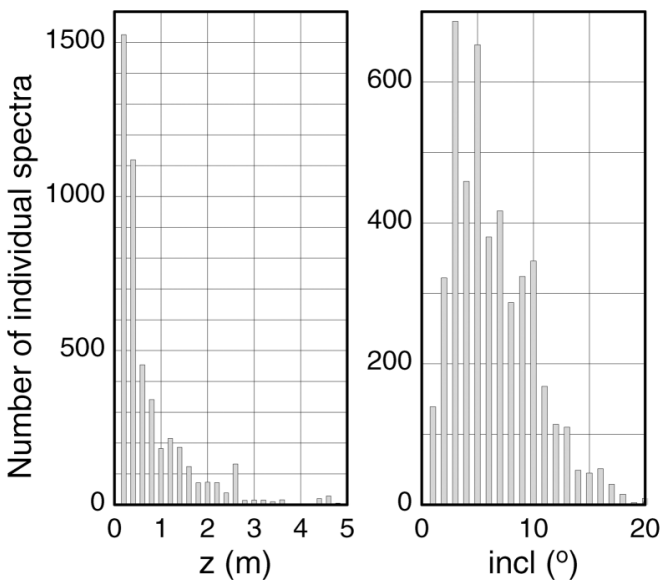

Fig. 1. Sensor depth and inclination of all individual data takes.

modeling than using the pressure sensor (which has just $0.1 \mathrm{~m}$ resolution), the $\operatorname{var}[z]$ value in Table 1 is from the $E_{d}$ fits as described below.

\section{Data Analysis}

The raw data of the irradiance sensor were calibrated, resampled to the interval $350-900 \mathrm{~nm}$ in steps of $5 \mathrm{~nm}$, and converted to ASCII format using the TriOS software MSDA (Multi Sensor Data Acquisition, version 1.99, build 0214). The radiometric response of the $E_{d}$ sensor used for calibration has been determined in air by the instrument manufacturer in March 2003. Because refraction, reflection, and transmission at the entrance optics are different if the medium is water or air, the sensor response in water changes by the so-called immersion factor $I_{f}$ compared to that in air [24]. Zibordi and Darecki [25] have determined this factor for three TriOS RAMSES-ACC-VIS radiometers in the spectral range $400-700 \mathrm{~nm}$ and concluded that 1.376 is a convenient approximation at an uncertainty of $\sim 2 \%$. Hence, all irradiance spectra exported by MSDA were multiplied by $I_{f}=1.376$ during postprocessing.

Table 1. Model Parameters and Values Used for Simulation

\begin{tabular}{|c|c|c|}
\hline Parameter & Value & Description \\
\hline$f_{d d}$ & 1 & $\begin{array}{l}\text { Fraction of direct downwelling } \\
\text { irradiance }\end{array}$ \\
\hline$f_{d s}$ & 1 & $\begin{array}{l}\text { Fraction of diffuse downwelling } \\
\text { irradiance }\end{array}$ \\
\hline$z$ & $0.7 \mathrm{~m}$ & Depth \\
\hline$\theta_{\text {Sun }}$ & $40^{\circ}$ & Sun zenith angle \\
\hline$\theta_{s}$ & $0^{\circ}$ & Sensor tilt angle \\
\hline $\operatorname{var}\left[\Delta f_{d d} / f_{d d}\right]$ & 0.07 & $\begin{array}{l}\text { Variance of direct component } \\
\text { of irradiance }\end{array}$ \\
\hline $\operatorname{var}\left[\Delta f_{d s} / f_{d s}\right]$ & 0.11 & $\begin{array}{l}\text { Variance of diffuse component } \\
\text { of irradiance }\end{array}$ \\
\hline $\operatorname{var}[z]$ & $0.003 \mathrm{~m}^{2}$ & Variance of sensor depth \\
\hline $\operatorname{var}\left[\theta_{s}\right]$ & $0.0007 \mathrm{sr}^{2}$ & Variance of sensor tilt angle \\
\hline$C$ & $2 \mathrm{mgm}^{-3}$ & Phytoplankton concentration \\
\hline$X$ & $0.6 \mathrm{gm}^{-3}$ & Suspended matter concentration \\
\hline$Y$ & $0.3 \mathrm{~m}^{-1}$ & Gelbstoff absorption at $440 \mathrm{~nm}$ \\
\hline
\end{tabular}

The equations derived in Section 2 and the model parameters summarized in Table 1 are used in Section $\underline{4}$ to simulate the spectral dependency of the factors responsible for irradiance variance and to illustrate the dependencies of $r_{d}$ and $\left[r_{d} /\left(r_{d}+\right.\right.$ 1) $]^{2}$ on the wavelength, Sun zenith angle, and depth.

The depth-dependent model of downwelling irradiance, as given by the equations in Subsections 2.A and 2.D, was implemented into the public domain software WASI $[26,27]$, which allows simulation of different types of optical measurements in aquatic environments and analyze such measurements by inverse modeling (see [11] for more details). Inverse modeling was applied to all 4375 single spectra $E_{d, i}(\lambda)$ in order to determine the parameters $f_{d d, i}$, $f_{d s, i}, z_{i}, X_{i}$, and $Y_{i}$ for each individual data take $i$. $\rho_{d d}$ and $\rho_{d s}$ were calculated as a function of $\theta_{\text {Sun }}$, and $n_{w}=1.33$ was set to represent fresh water. The $X$ and $Y$ values of Table 1 are the resulting averages of all measurements with a water column of at least $1.5 \mathrm{~m}$ above the sensor [11].

The variability of $f_{d d}, \overline{f_{d s}}$, and $z$ during a measurement and the resulting impact on irradiance was analyzed as follows. First, the mean values of $f_{d d}$, $f_{d s}$, and $z$ were calculated for each measurement by averaging the values $f_{d d, i}, f_{d s, i}$, and $z_{i}$ of the related individual data takes. Then the differences between the individual parameters and the means were calculated as $\Delta f_{d d, i}=f_{d d, i}-f_{d d}, \Delta f_{d s, i}=f_{d s, i}-$ $f_{d s}$, and $\Delta z_{i}=z_{i}-z$. According to Eq. (11), the relevant parameters of irradiance variance are the relative changes of $f_{d d}$ and $f_{d s}$ and the absolute changes of $z$. Thus, the analysis of variability in Section 5 is based on the parameters $\Delta f_{d d . i} / f_{d d}$, $\Delta f_{d s, i} / f_{d s}$, and $\Delta z_{i}$. Furthermore, the correlation coefficients between these parameters and the corresponding irradiance changes are calculated for selected depth intervals to investigate their impact on irradiance. The average variances $\operatorname{var}\left[\Delta f_{d d} / f_{d d}\right]$, $\operatorname{var}\left[\Delta f_{d s} / f_{d s}\right], \operatorname{var}[z]$ listed in Table 1 were obtained by calculating the variances of $\Delta f_{d d, i} / f_{d d}, \Delta f_{d s, i} / f_{d s}$, and $\Delta z_{i}$ of all 421 measurements and then averaging these.

In order to quantify spectral changes during a measurement, two indices are introduced, as follows:

$$
\begin{aligned}
& \gamma_{\mathrm{VIS}, i}=\frac{E_{d, i}(400)}{E_{d}(400)}-\frac{E_{d, i}(700)}{E_{d}(700)}, \\
& \gamma_{\mathrm{NIR}, i}=\frac{E_{d, i}(755)}{E_{d}(755)}-\frac{E_{d, i}(700)}{E_{d}(700)} .
\end{aligned}
$$

The index $\gamma_{\text {VIS }}$ is a measure of spectral changes across the visible spectral range from 400 to $700 \mathrm{~nm}$; the index $\gamma_{\text {NIR }}$ is a measure of changes in the near infrared between 700 and $755 \mathrm{~nm}$. Two indices are used, because it is expected from Eq. (11) that basically two variable parameters influence the spectral shape of irradiance: geometry and depth. The figure of $700 \mathrm{~nm}$ is selected as common reference wavelength because absorption of water is relatively low 
below $700 \mathrm{~nm}$ and increases strongly above $700 \mathrm{~nm}$; hence, geometry effects are expected to dominate irradiance changes below $700 \mathrm{~nm}$ and changes of the sensor depth above. Both indices can be derived directly from the measurements $E_{d, i}(\lambda)$ without the necessity of fitting a model to the spectrum; as this is the case for $f_{d d, i}, f_{d s, i}$, and $z_{i}$.

\section{Model Results}

\section{A. Sources of Irradiance Variance}

Changes of the downwelling irradiance during a measurement can be caused by variable fractions of direct and diffuse radiation $\left(f_{d d}, f_{d s}\right)$ due to the wave focusing effect, by a variable sensor tilt angle $\left(\theta_{s}\right)$, and by a variable sensor depth $(z)$. Equation (11) describes quantitatively the impact of these factors on the irradiance variance. It shows that each effect changes an undisturbed spectrum $E_{d}(\lambda)$ by a characteristic wavelength-dependent function, except variable $f_{d d}$ and $\theta_{s}$, which induce the same spectral change. These characteristic functions are illustrated in Fig. 2. The shapes of the curves show the spectral changes of irradiance induced by environmental and experimental conditions, and the magnitudes represent the average contribution of each effect to total irradiance variance for our dataset. The simulations were performed using the values of Table 1 .

The dominating factor of irradiance variance in the wavelength range from 410 to $830 \mathrm{~nm}$ are fluctuations of the direct component due to wave focusing (Fig. 2 , curve 1). A tilting sensor induces the same wavelength dependent variance (curve 2), but the effect is 2 orders of magnitude lower. Curve 1 was calculated as $0.07\left[r_{d} /\left(r_{d}+1\right)\right]^{2}$, where 0.07 is the average variance of $\Delta f_{d d} / f_{d d}$ of all our measurements; curve 2 was calculated as $0.0007\left[r_{d} /\left(r_{d}+1\right)\right]^{2} \tan ^{2} \theta^{\prime}$ Sun, where $0.0007 \mathrm{sr}^{2}$ corresponds to a $1.6^{\circ}$ standard deviation of sensor inclination. The variance of diffuse irradiance is shown as curve 3 . It was calculated as $0.11 /\left(r_{d}+1\right)^{2}$, with 0.11 expressing the average var-

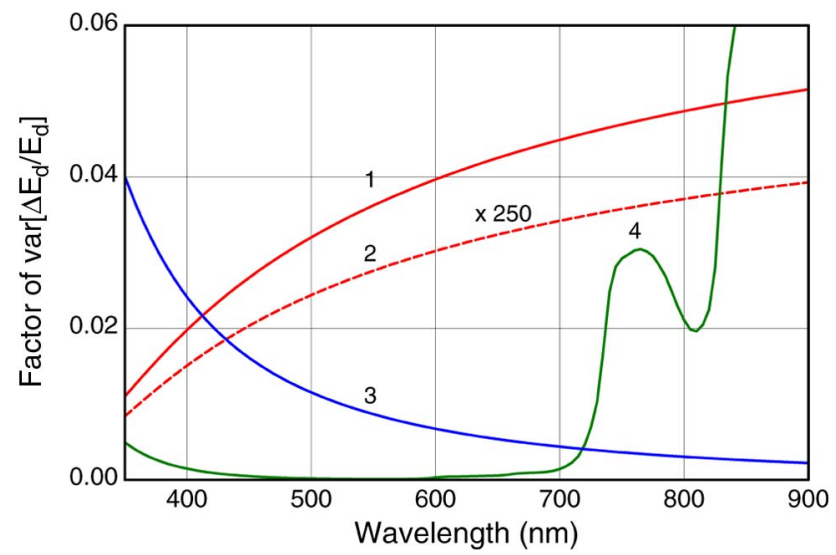

Fig. 2. (Color online) Environmental and experimental factors responsible for irradiance variance: 1 , variance of direct irradiance due to wave focusing; 2 , variance induced by tilting sensor (scaled by a factor of 250); 3 , variance of diffuse irradiance due to wave focusing; 4, variance of sensor depth. iance of $\Delta f_{d s} / f_{d s}$ for our measurements. Curve 4 shows the impact of variable sensor depth on spectral irradiance. It was calculated as $0.003\left[\left(K_{d d} r_{d}\right)\right.$ $\left.\left.\cos \theta_{\text {Sun }}^{\prime}+1.18 K_{d s}\right) /\left(r_{d}+1\right)\right]^{2}$, where the variance of $0.003 \mathrm{~m}^{2}$ represents a $5.5 \mathrm{~cm}$ standard deviation of the sensor depth.

\section{B. Ratio of Direct to Diffuse Irradiance}

When waves modulate the water surface or when the sensor is tilted, the relative intensities of the direct and diffuse components of irradiance are altered. The resulting changes of the ratio $r_{d}$ of direct-todiffuse irradiance are a major cause of spectral changes of the measured downwelling irradiance. This fact is expressed quantitatively by Eq. (11), where $r_{d}$ determines the intensity of each factor contributing to $E_{d}$ variability during a measurement. Figure 3 illustrates the dependency of $r_{d}$ on the wavelength, Sun zenith angle, and depth. The wavelength dependency is almost linear close to the surface, but with increasing depth, the spectral features of the water attenuation get more and more pronounced in the infrared. The simulations were performed using the values of Table 1 , except $z=0$ was set for the $\theta_{\text {Sun }}$ series in order to illustrate $r_{d}\left(0_{-}\right)$ near the surface.

\section{Geometry Factor}

The geometry factor $\left[r_{d} /\left(r_{d}+1\right)\right]^{2}$ is the dominating characteristic function describing spectral changes of $E_{d}$. This is clearly demonstrated by Fig. 2 , where the spectral shape of the dominating curve 1 is given by $\left[r_{d} /\left(r_{d}+1\right)\right]^{2}$. Figure 4 illustrates the dependency of the geometry factor on wavelength, Sun zenith angle, and sensor depth. The simulations were performed using the values of Table 1 , except $z=0$ was set for the $\theta_{\text {Sun }}$ series in order to illustrate the geometry factor near the surface.

\section{Experimental Results}

\section{A. Sources of Variability During a Measurement}

Typical examples for the observed variability between individual data takes of a measurement are given in Figs. 5 and 6.

The measurement shown in Fig. 5 was performed on 29 July 2003, 15:05 h local time, at Lake Constance (station RE17). Cloud cover was $4 / 8$. Nineteen single spectra were recorded during a measurement time of $100 \mathrm{~s}$. Each individual spectrum was fitted using Eq. (1) with $z, f_{d d}$, and $f_{d s}$ as fit parameters; for the other model parameters, the values from Table 1 were taken. The result was $z=0.0012 \pm$ $0.010 \mathrm{~m}, \quad f_{d d}=0.77 \pm 0.28, \quad f_{d s}=0.92 \pm 0.03$. Because sensor depth was very stable, the variability of irradiance is caused primarily by the wave focusing effect. The variance has the spectral shape that is expected from curve 1 of Fig. 2.

The measurement shown in Fig. $\underline{6}$ was performed on 30 July $2004,12: 40 \mathrm{~h}$ local time, at Lake Constance (station 20_1). Cloud cover was $2 / 8$. Fitting 


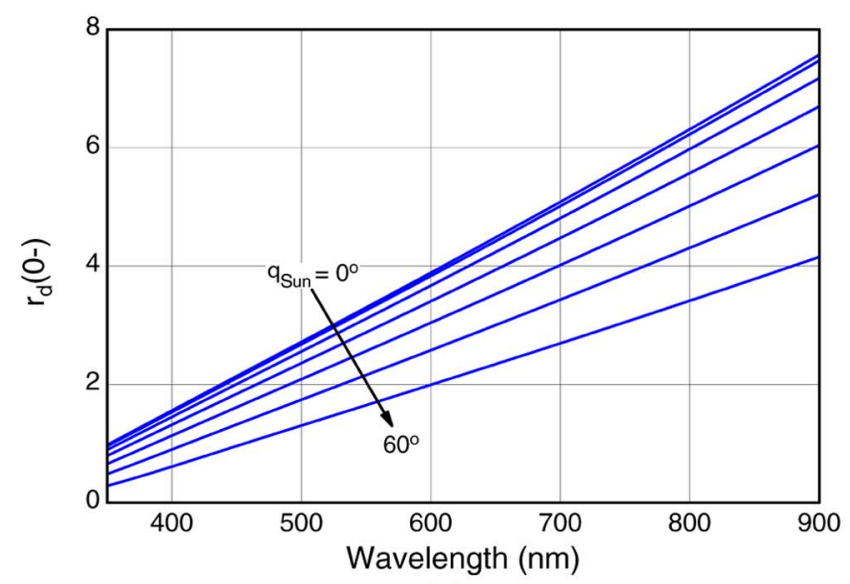

(a)

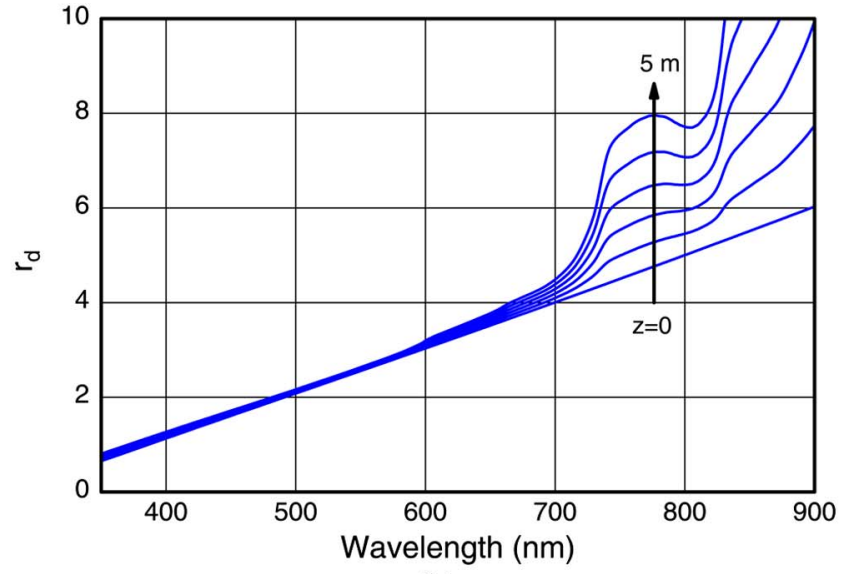

(b)

Fig. 3. (Color online) Dependency of the ratio of direct to diffuse irradiance on (a) Sun zenith angle and (b) depth.

of the individual spectra resulted in $z=0.061 \pm$ $0.062 \mathrm{~m}, f_{d d}=1.06 \pm 0.13$, and $f_{d s}=1.12 \pm 0.14$. Compared to the example before, $f_{d d}$ is more stable, but $z$ is much more variable. Consequently, the spectral variability of irradiance is dominated by variable sensor depth. Comparison with curve 4 of Fig. 2 shows that the variance has the expected spectral shape.

Visual screening of all measurements revealed a similarity of each spectrum $\operatorname{var}\left[\Delta E_{d} / E_{d}\right]$ either to Fig. 5 or to Fig. 6 or to a combination of both. In order to underpin this observation statistically, principal component analysis was performed for the variances $\operatorname{var}\left[\Delta E_{d} / E_{d}\right]$ of all 421 measurements using the function "prcomp" of the software R [28]. Figure 7 shows the result.

The first four components explain $98 \%$ of the irradiance variance. The dominating component, responsible for $85.5 \%$ of the variance (curve 1 ), is spectrally flat, except for a slight curvature at the borders of the spectral range that may be caused by calibration errors (detector nonlinearity for low signals, wavelength-dependency of immersion factor). Consequently, the main impact of changing environmental and experimental conditions on downwelling irradiance is an altered scaling factor, i.e., a change of intensity.

The second principal component, responsible for $6.2 \%$ of the variance for the current dataset, shows the typical spectral feature of the sensor depth variation (curve 4 of Fig. 2), which is caused by water attenuation. The third component, responsible in our dataset for $4.7 \%$ of the variance, is similar to the spectral shape of the geometry factor $\left[r_{d} /\left(r_{d}+\right.\right.$ 1) $]^{2}$ (curves 1 and 2 of Fig. 2 ); i.e., it is caused by variable geometric conditions. The fourth component, accounting for $1.6 \%$ of the variance, cannot be clearly assigned to one of the factors of Fig. 2 , but it resembles a combination of components 2 and 3 . The variance of diffuse irradiance (curve 3 of Fig. 2) cannot be identified clearly in the first four components. All further components contribute less than $1 \%$ to the total variance.

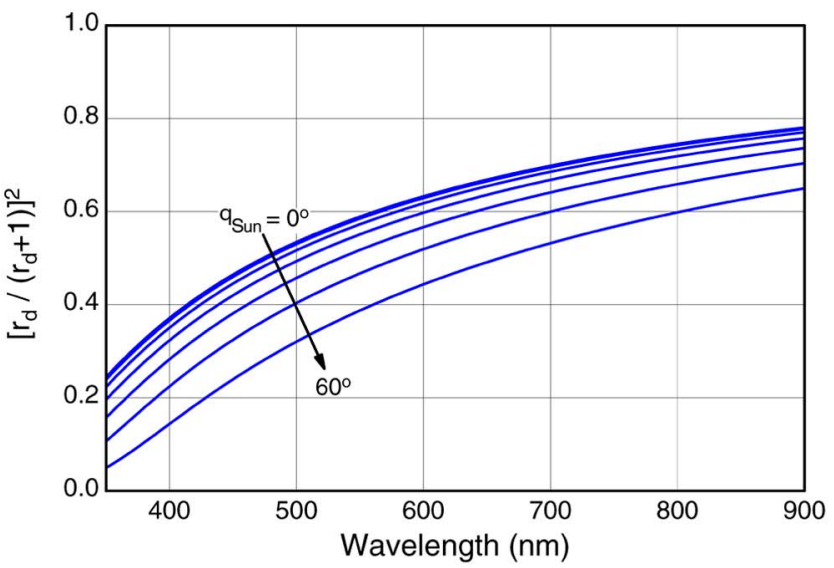

(a)

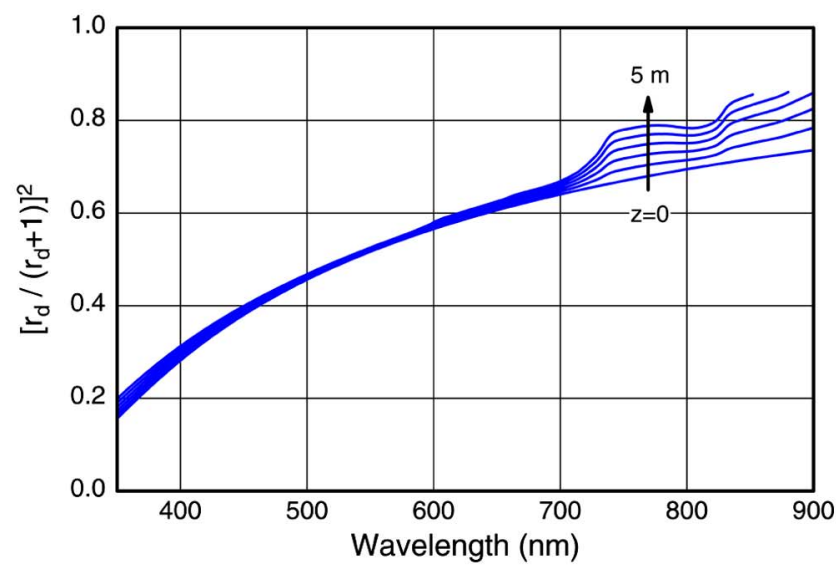

(b)

Fig. 4. (Color online) Illustration of the geometry factor. 


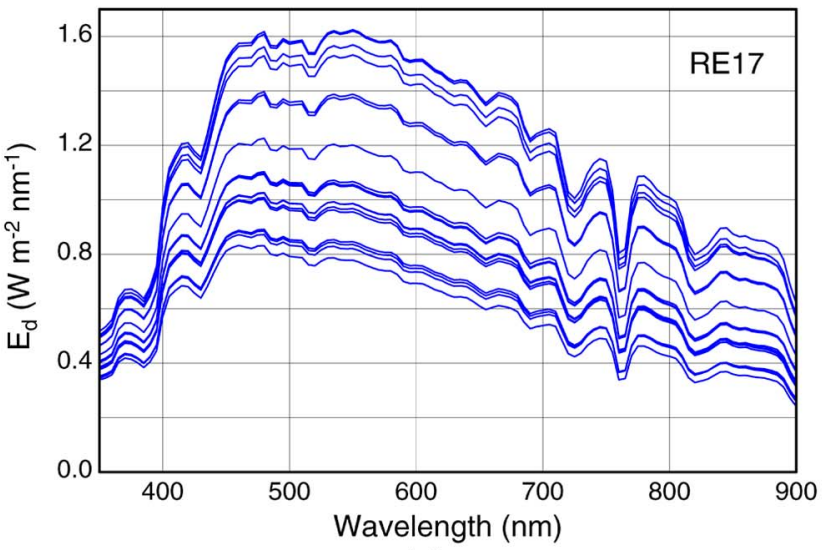

(a)

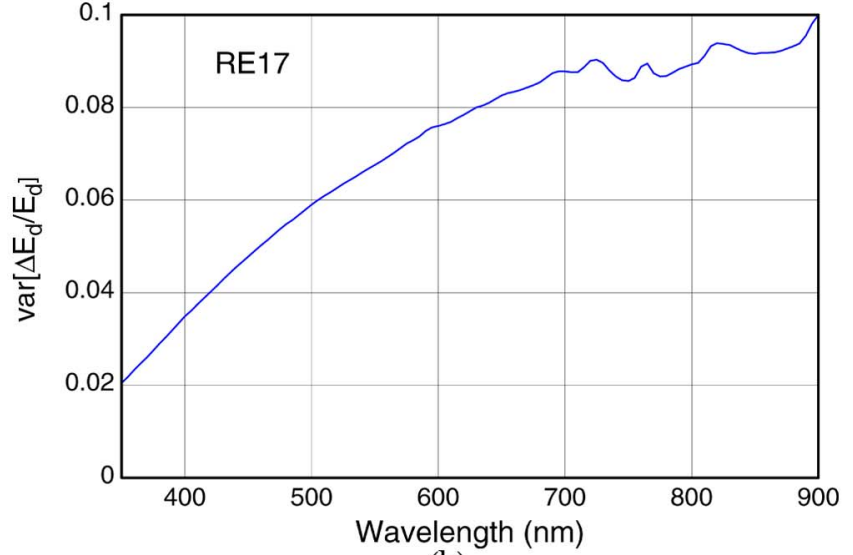

(b)

Fig. 5. (Color online) Example for the variability of irradiance measurements caused mainly by wave focusing and changing sensor tilt.

\section{B. Intensity Variability}

We express intensity variability of irradiance during a measurement as spectral average of $\Delta E_{d, i} / E_{d}$ from 400 to $800 \mathrm{~nm}$, because for these wavelengths, the principal component of $\operatorname{var}\left[\Delta E_{d} / E_{d}\right]$ is constant (see curve 1 of Fig. 7). $\Delta E_{d, i} / E_{d}=\left(E_{d, i}-E_{d}\right) / E_{d}$ denotes the relative difference of a single spectrum $E_{d, i}$ compared to the average $E_{d}$ of all spectra collected during the measurement. Figure 8 shows these relative intensity changes for all $43 \overline{75}$ single spectra as a function of depth and also the resulting variances of the 421 measurements. It can be seen that variability can be very large near the surface, and it decreases with depth.

The depth dependency of the irradiance intensity variability is summarized quantitatively in Table 2. Variability is maximal for the depth range of 0.5 to $1 \mathrm{~m}$, in which the average variance $\Delta E_{d} / E_{d}$ is 0.075 , corresponding to a relative standard deviation of $27 \%\left(N^{\prime}=46\right)$. Above and below that depth range, variability is on average lower, with relative standard deviations in the order of $18 \%$. The gray line in Fig. 8 illustrates the depth-averaged $\operatorname{var}\left(\Delta E_{d} / E_{d}\right)$

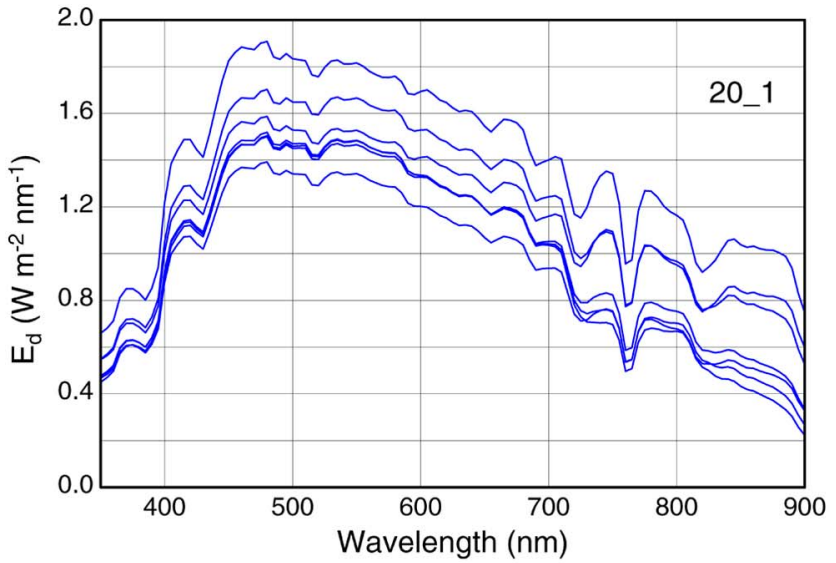

(a) values of Table $\underline{2}$. It makes the peak of the irradiance variance between 0.5 to $1 \mathrm{~m}$ evident.

As was described in Subsection 3.B, the integration time $t$ was set automatically by the instrument electronics. The impact of this variable integration time on the measured irradiance variance was studied by correlation analysis. The correlation coefficient, $r$, is 0.29 between $t$ and $z$ for the range 0.1 to $5 \mathrm{~m}(N=3026)$, which is significant on the $99 \%$ confidence level, but explains only $8 \%$ of the $t$ variability. Hence, it is not surprising that the correlation between $t$ and $\operatorname{var}\left[\Delta E_{d} / E_{d}\right]$ is very low $(r=-0.028)$ and not significant. Consequently, the observed decrease of irradiance variance with depth is not caused by an extended integration time.

In order to investigate the causes of intensity variability as a function of depth, Table $\underline{2}$ shows the coefficient of determination, $r^{2}$, for the sources of intensity variability at different depth intervals. Calculation of $\Delta f_{d d} / f_{d d}, \Delta f_{d s} / f_{d s}$, and $\Delta z$ was described in Subsection 3.C. The last three lines of Table 2 show that the correlation between these parameters is in general low, suggesting that Eq. (1) is a useful model with independent parameters. The

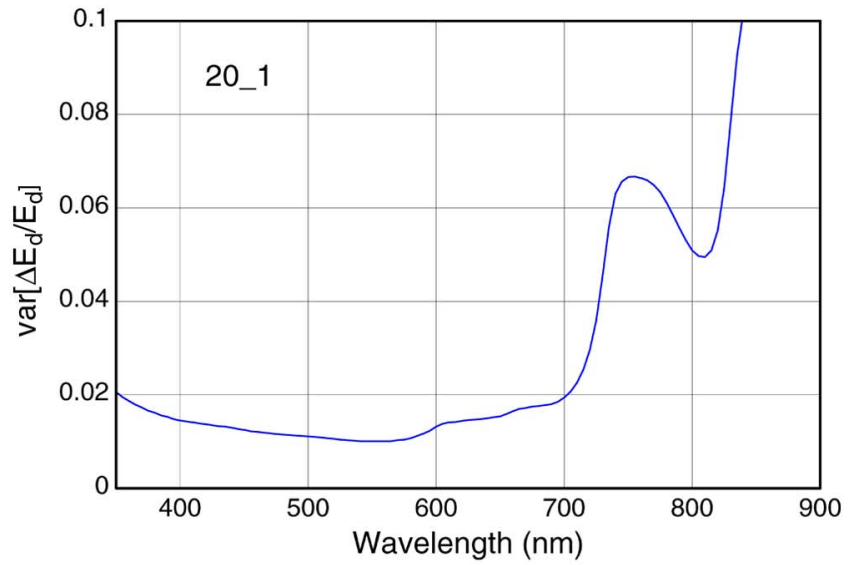

(b)

Fig. 6. (Color online) Example for the variability of irradiance measurements caused mainly by sensor depth variation. 


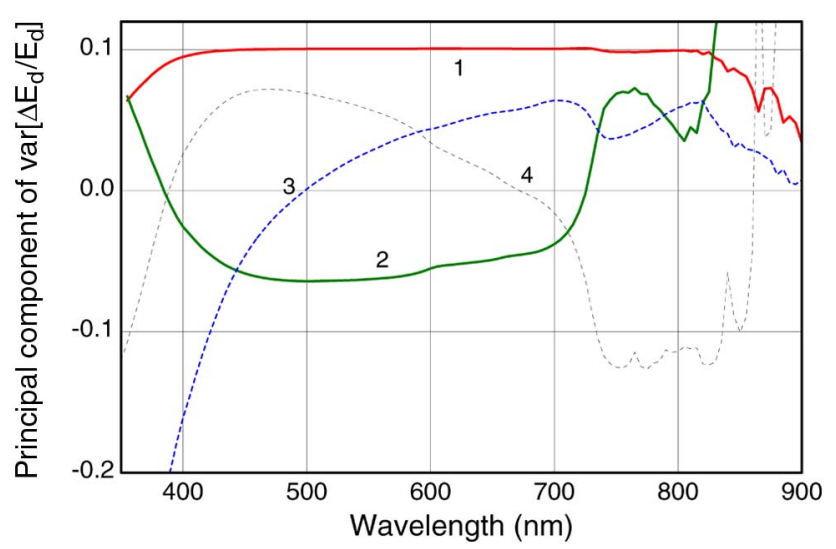

Fig. 7. (Color online) Dominating components of irradiance variance. Proportions of variance: $1=85.5 \%, 2=6.2 \%, 3=4.7 \%$, $4=1.6 \%$.

correlation is increased, with $r^{2}$ values from 0.02 to 0.16 , between $\Delta f_{d d} / f_{d d}$ and $\Delta f_{d s} / f_{d s}$, indicating a weak relationship between the variabilities of direct and diffuse irradiance due to their common origin, the wave-roughened water surface.

Looking at the correlations with $\Delta E_{d} / E_{d}$, it can be concluded that fluctuations of direct irradiance $\left(\Delta f_{d d} / f_{d d}\right)$ are the dominating factor of intensity variability, explaining up to $82 \%$ of variance. The second rank has a variable sensor depth $(\Delta z)$, which gets more and more important with depth; it explains up to $59 \%$ of irradiance variability (for the depth range 2.5 to $3.0 \mathrm{~m}$ ). The variability of the diffuse irradiance $\left(\Delta f_{d s} / f_{d s}\right)$ is of no importance at all depths. Figure $\underline{9}$ illustrates this result at two examples: it shows the high correlation between $\Delta E_{d} / E_{d}$ and $\Delta f_{d d} / f_{d d}$ near the surface and between $\Delta E_{d} / E_{d}$ and $\Delta z$ for the depth range of 2.5 to $3.0 \mathrm{~m}$.

\section{Spectral Variability}

Typical spectral changes of irradiance are illustrated in Fig. 10 for the measurements RE17 and 20_1, which were already shown above in Figs. $\underline{5}$ and $\underline{6}$.
The individual spectra $E_{d, i}(\lambda)$ were normalized by the measurements' mean $E_{d}(\lambda)$, and these ratios were normalized at $700 \mathrm{~nm}$.

Variability of irradiance during the measurement RE17 was caused primarily by geometry effects, while that of 20_1 originated mainly from depth variation (see Subsection 5.A). In the first case, the spectral shape of $E_{d}(\bar{\lambda})$ is affected especially in the visible, in the second case, mostly in the near infrared. The indices $\gamma_{\text {VIS }}$ and $\gamma_{\text {NIR }}$ defined in Subsection 3.C are a measure of these spectral changes. $\gamma_{\mathrm{VIS}}$ corresponds to the difference $y(400)-1, \gamma_{\text {NIR }}$ to $y(755)-1$ where $y$ denotes the ordinate of Fig. 10. $\gamma_{\text {VIS }}$ ranges from -0.12 to 0.19 for $\mathrm{RE} 17$ and from -0.04 to 0.06 for $20 \_1$; the means of the absolute values are 0.09 and 0.02 , respectively. $\gamma_{\mathrm{NIR}}$ ranges from -0.02 to 0.01 for RE17 and from -0.18 to 0.18 for $20 \_1$ with the corresponding means of 0.01 and 0.11 . These indices $\gamma_{\mathrm{VIS}}$ and $\gamma_{\mathrm{NIR}}$ were calculated for all 4375 single spectra of our dataset. The result is shown in Fig. 11.

It can be seen that spectral variability during a measurement can be quite significant, both across the visible spectral range and in the near infrared. Table 3 summarizes the mean absolute values as function of depth. $\gamma_{\text {VIS }}$ does not depend that much on depth; the average of all values is 0.054. $\gamma_{\mathrm{NIR}}$ changes at a depth of $1 \mathrm{~m}$ : the average is 0.057 for the range of 0.1 to $1.0 \mathrm{~m}$ and 0.037 for depths below $1 \mathrm{~m} .20 \%$ of our data exhibits spectral changes of more than $10 \%$ either in the visible or in the near infrared.

Table 3 summarizes further some statistical relationships for the spectral indices. The correlation between $\gamma_{\text {VIS }}$ and $\gamma_{\text {NIR }}$ is very low at all depths; i.e., $\gamma_{\text {VIS }}$ and $\gamma_{\text {NIR }}$ are sensitive to two basically independent effects. $\gamma_{\mathrm{VIS}}$ is highly correlated to $\Delta f_{d d} / f_{d d}$ and significantly to $\Delta f_{d s} / f_{d s}$; the respective coefficients of determination are $r^{2}=0.64$ and $r^{2}=0.15$ for all data from 0.1 to $5 \mathrm{~m}(N=3449)$. Hence, the index $\gamma_{\mathrm{VIS}}$ is sensitive to the wave focusing effect. $\gamma_{\mathrm{NIR}}$ is not

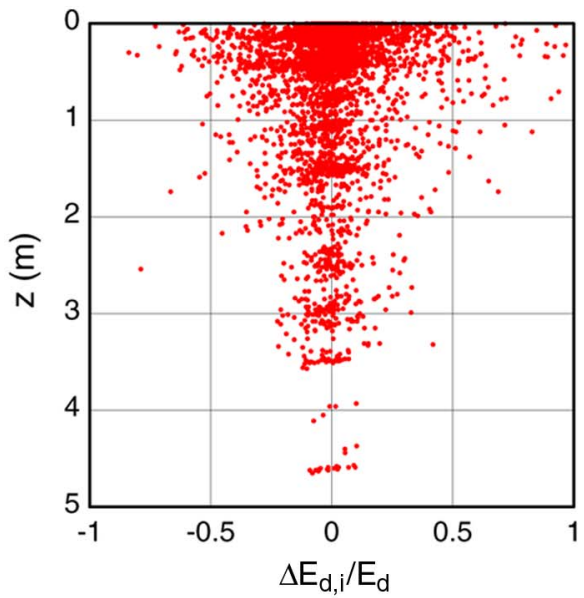

(a)

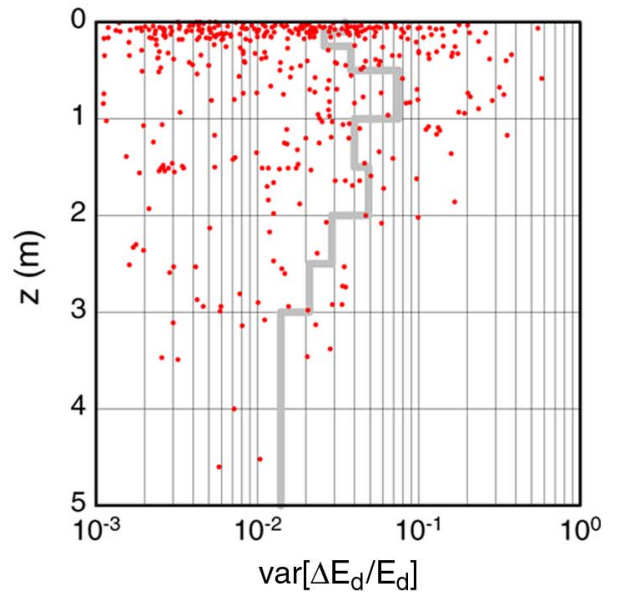

(b)

Fig. 8. (Color online) Intensity variability of irradiance as a function of depth: (a) relative differences of a single spectra and (b) variances during a measurement. 
Table 2. Depth Dependency of Intensity Variability and Correlation with Main Causes ${ }^{a}$

\begin{tabular}{|c|c|c|c|c|c|c|c|c|}
\hline Depth Range (m) & $0.10-0.25$ & $0.25-0.50$ & $0.5-1.0$ & $1.0-1.5$ & $1.5-2.0$ & $2.0-2.5$ & $2.5-3.0$ & $3.0-5.0$ \\
\hline $\operatorname{var}\left(\Delta E_{d} / E_{d}\right)$ & 0.026 & 0.038 & 0.075 & 0.040 & 0.049 & 0.029 & 0.021 & 0.014 \\
\hline$r^{2}\left(\Delta E_{d} / E_{d}, \Delta f_{d s} / f_{d s}\right)$ & 0.00 & 0.03 & 0.00 & 0.00 & 0.02 & 0.04 & 0.01 & 0.00 \\
\hline$r^{2}\left(\Delta E_{d} / E_{d}, \Delta z\right)$ & 0.08 & 0.04 & 0.13 & 0.22 & 0.28 & 0.32 & 0.59 & 0.34 \\
\hline$r^{2}\left(\Delta f_{d d} / f_{d d}, \Delta f_{d s} / f_{d s}\right)$ & 0.05 & 0.02 & 0.11 & 0.15 & 0.16 & 0.13 & 0.07 & 0.08 \\
\hline
\end{tabular}

${ }^{a} N$, number of single spectra; $N^{\prime}$, number of measurements. See text for details.

noteworthily related with $\Delta f_{d d} / f_{d d}$ and $\Delta f_{d s} / f_{d s}$ at any depth, but highly correlated to $\Delta z$ with $r^{2}=$ 0.68 in the upper meter $(N=2445)$. The correlation decreases further down. Visual inspection of the measurements showed that sensor noise becomes significant at depths above approximately $2 \mathrm{~m}$ for wavelengths in the range of 735 to $900 \mathrm{~nm}$. This may explain the low correlation between $\gamma_{\mathrm{NIR}}$ and $\Delta z$ for depths $>2 \mathrm{~m}$.

\section{Summary}

In situ measurements of downwelling irradiance in water $\left(E_{d}\right)$ are in general highly disturbed by large intensity variations that are accompanied by

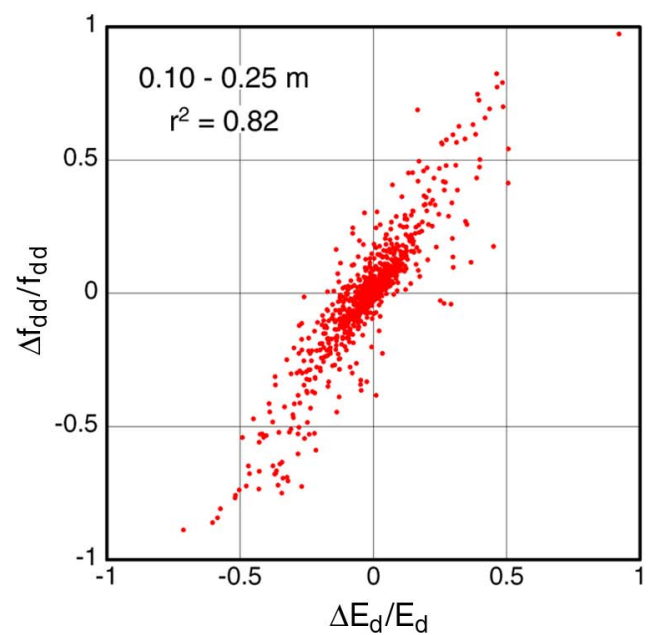

(a)

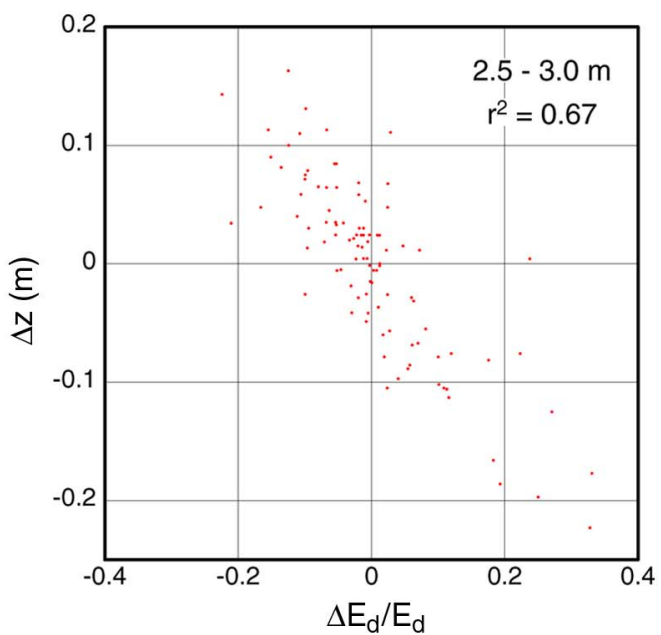

(b)

Fig. 9. (Color online) Relationship between (a) irradiance intensity variability and the changes of direct component and (b) sensor depth for selected depth intervals.

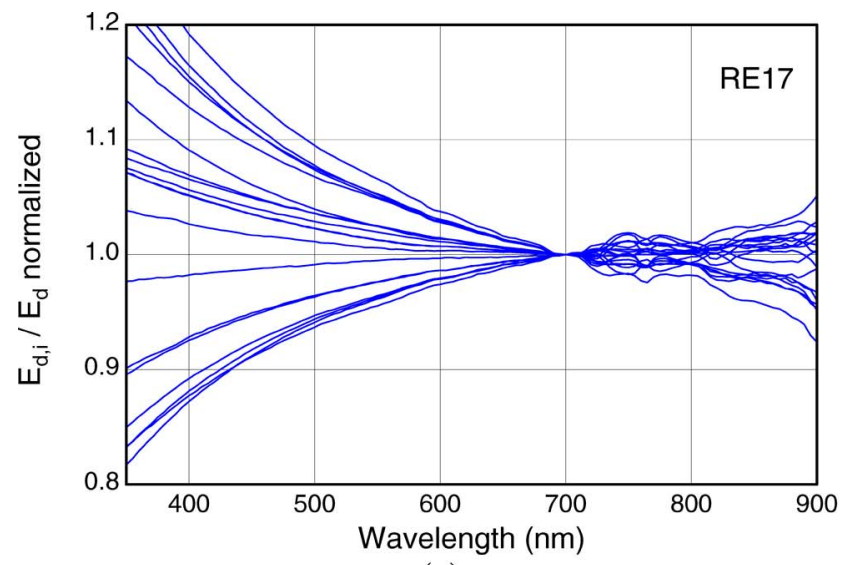

(a)

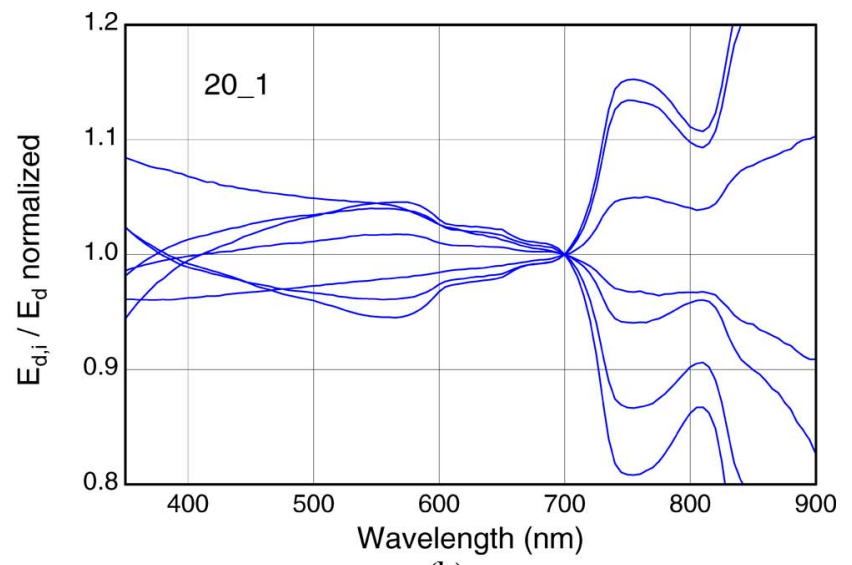

(b)

Fig. 10. (Color online) Examples for spectral variability during a measurement: (a) RE17, relative irradiance changes caused mainly by wave focusing and changing sensor tilt and (b) 20_1, caused mainly by depth variation. 


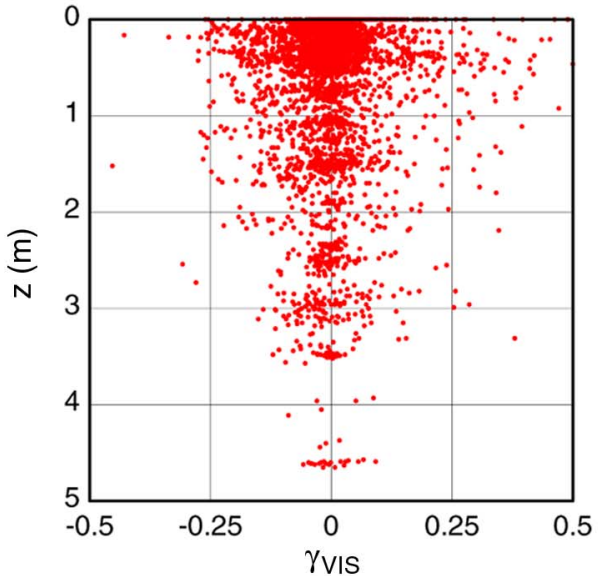

(a)

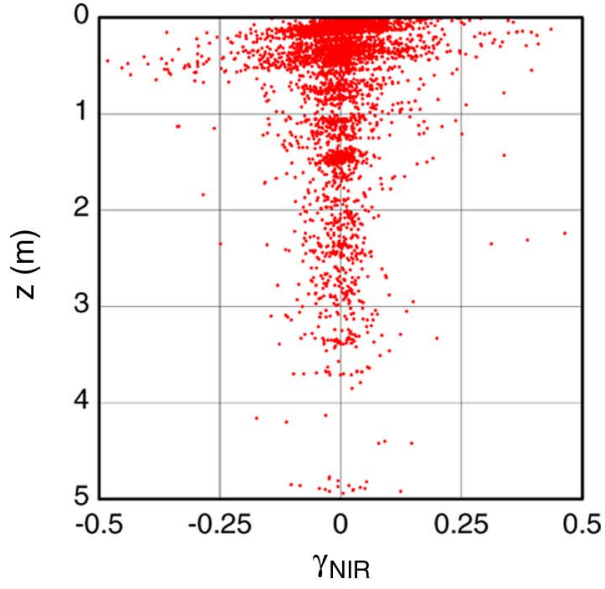

(b)

Fig. 11. (Color online) Spectral variability of irradiance as function of depth: (a) relative spectral changes across the visible (400 versus $700 \mathrm{~nm})$ and $(b)$ in the near infrared (755 versus $700 \mathrm{~nm})$.

changes of spectral shape. We derived an equation [Eq. (11)] that relates irradiance variance to changing environmental and measurement conditions. An environmental factor that is well known to induce very strong short-term intensity variations is the wave focusing effect. We introduced two parameters, $f_{d d}$ and $f_{d s}$, to quantify the actual fractions of the direct and diffuse components of irradiance. The environmental condition responsible for $E_{d}$ variance, the wave-roughened water surface, is then parameterized by the variances of $f_{d d}$ and $f_{d s}$ to describe the wave focusing effect and by the variance of sensor depth to describe wave-induced changes in the water column's height above the sensor. Additionally, the measurement conditions are defined by the sensor tilt angle $\left(\theta_{s}\right)$, sensor depth $(z)$, and by the variance of $\theta_{s}$. Hence, Eq. (11) is a model that attributes the variance of $E_{d}$ intensity and spectral shape to the parameters $f_{d d}, f_{d s}, \theta_{s}$, and $z$ and to their variances. The equation shows that the ratio of direct-to-diffuse irradiance, $r_{d}$, plays a key role for spectral changes of $E_{d}$. It shows further that a variable direct component of irradiance induces the same spectral change as a tilted or swaying sensor, which are both proportional to $\left[r_{d} /\left(r_{d}+1\right)\right]^{2}$ (geometry factor). The dependency of $r_{d}$ and $\left[r_{d} /\left(r_{d}+1\right)\right]^{2}$ on wavelength, depth, and Sun zenith angle was illustrated.

A large dataset was collected during field campaigns in three German lakes at sensor depths between 0 and $5 \mathrm{~m}$. It consists of 421 measurements, each composed of 4 to 50 single spectra of downwelling irradiance. The measurements were first analyzed statistically using principal component analysis to determine the number and spectral shapes of the major factors responsible for $E_{d}$ variability and to validate the irradiance variance model. The first component, explaining $85 \%$ of irradiance variance, is spectrally flat, i.e., changing environmental and experimental conditions affect primarily $E_{d}$ intensity. The spectral shapes of the two next relevant components are similar to those expected from the model for variable sensor depth and changing geometry. Statistically relevant components with spectral shapes inconsistent to the model were not observed, suggesting that the model accounts for all relevant sources of irradiance variability.

The basis of the irradiance variance model is an $E_{d}$ model that distinguishes between the direct and diffuse components of irradiance [11]. This $E_{d}$ model was used to determine the parameters $f_{d d}, f_{d s}$, and $z$ for all single measurements by inverse modeling.

Table 3. Depth Dependency of Indices Describing Spectral Variability of Irradiance and Correlation with Parameters Describing the Main Causes ${ }^{a}$

\begin{tabular}{ccccccccc}
\hline Depth Range $(\mathrm{m})$ & $0.10-0.25$ & $0.25-0.50$ & $0.5-1.0$ & $1.0-1.5$ & $1.5-2.0$ & $2.0-2.5$ & $2.5-3.0$ & $3.0-5.0$ \\
\hline$N / N^{\prime}$ & $896 / 81$ & $1051 / 60$ & $492 / 46$ & $367 / 39$ & $209 / 28$ & $123 / 10$ & $128 / 19$ & $144 / 11$ \\
$<\left|\gamma_{\mathrm{VIS}}\right|>$ & 0.046 & 0.052 & 0.071 & 0.059 & 0.069 & 0.052 & 0.051 \\
$<\left|\gamma_{\mathrm{NIR}}\right|>$ & 0.051 & 0.059 & 0.066 & 0.040 & 0.031 & 0.032 & 0.038 & 0.042 \\
$r^{2}\left(\gamma_{\mathrm{VIS}}, \gamma_{\mathrm{NIR}}\right)$ & 0.01 & 0.01 & 0.00 & 0.00 & 0.01 & 0.01 & 0.08 & 0.04 \\
$r^{2}\left(\gamma_{\mathrm{VIS}}, \Delta f_{d d} / f_{d d}\right)$ & 0.65 & 0.63 & 0.56 & 0.72 & 0.77 & 0.73 & 0.84 \\
$r^{2}\left(\gamma_{\mathrm{VIS}}, \Delta f_{d s} / f_{d s}\right)$ & 0.22 & 0.18 & 0.14 & 0.25 & 0.13 & 0.16 & 0.05 \\
$r^{2}\left(\gamma_{\mathrm{VIS}}, \Delta z\right)$ & 0.01 & 0.01 & 0.05 & 0.13 & 0.07 & 0.25 & 0.24 & 0.17 \\
$r^{2}\left(\gamma_{\mathrm{NIR}}, \Delta f_{d d} / f_{d d}\right)$ & 0.00 & 0.00 & 0.00 & 0.00 & 0.03 & 0.01 & 0.11 \\
$r^{2}\left(\gamma_{\mathrm{NIR}}, \Delta f_{d s} / f_{d s}\right)$ & 0.01 & 0.00 & 0.00 & 0.00 & 0.01 & 0.02 & 0.00 \\
$r^{2}\left(\gamma_{\mathrm{NIR}}, \Delta z\right)$ & 0.74 & 0.74 & 0.62 & 0.32 & 0.14 & 0.02 & 0.00 & 0.11 \\
\hline
\end{tabular}

${ }^{a} N$, number of single spectra; $N^{\prime}$, number of measurements. 
Correlation with the corresponding $E_{d}$ intensities revealed that the dominating source of intensity fluctuations is the variability of the direct component and below $1 \mathrm{~m}$ additionally changes of sensor depth. Intensity fluctuations induced by variable sensor tilt angle are more than 2 orders of magnitude lower. The diffuse component's variability has a negligible influence on $E_{d}$ intensity. The relative standard deviation of $E_{d}$ intensity was typically in the order of $18 \%$, but it was increased to $27 \%$ in the depth range of 0.5 to $1 \mathrm{~m}$.

Spectral variability was investigated using two indices that measure the relative change of spectral shape between 400 and $700 \mathrm{~nm}$ and between 700 and $755 \mathrm{~nm}$. The index for the visible range depends only weakly on depth and reveals an average spectral variability of $5.4 \%$. The near-infrared index has a variability of $5.7 \%$ in the upper meter and $3.7 \%$ at larger depths. $20 \%$ of our data exhibits spectral changes above $10 \%$ either in the visible or in the near infrared. Comparison between the indices and the corresponding model parameters obtained from inverse modeling showed that in the visible, the wave focusing effect is the major cause of spectral changes, followed by depth variations, which get relevant below $2 \mathrm{~m}$. Spectral variability in the near infrared is always dominated by depth changes.

\section{References}

1. R. E. Walker, Marine Light Field Statistics (Wiley, 1994).

2. J. R. V. Zaneveld, E. Boss, and A. Barnard, "Influence of surface waves on measured and modeled irradiance profiles," Appl. Opt. 40, 1442-1449 (2001).

3. J. Dera and D. Stramski, "Maximum effects of sunlight focusing under a wind-disturbed sea surface," Oceanologia 23, 15-42 (1986).

4. J. Dera and D. Stramski, "Focusing of sunlight by sea surface waves: new results from the Black Sea," Oceanologia 34, 13-25 (1993).

5. V. L. Weber, "Coefficient of variation of underwater irradiance fluctuations," Radiophys. Quantum Electron. 53, 13-27 (2010).

6. D. A. Toole, D. A. Siegel, D. W. Menzies, M. J. Neumann, and R. C. Smith, "Remote-sensing reflectance determinations in the coastal ocean environment: impact of instrumental characteristics and environmental variability," Appl. Opt. 39, 456-469 (2000).

7. M. Lawson, B. Leavitt, N. Emanuel, R. Perk, J. Keck, and M. Hauschild, "Compensating for irradiance fluxes when measuring the spectral reflectance of corals in situ," GISci. Remote Sens. 43, 111-127 (2006).

8. H. Hofmann, A. Lorke, and F. Peeters, "Wave-induced variability of the underwater light climate in the littoral zone," Verh. Internat. Verein. Limnol. 30, 627-632 (2008).

9. H. Siegel and H.-J. Brosin, "Regional differences in the spectral reflectance of sea water," Beiträge zur Meereskunde 55, 71-77 (1986).
10. J.-M. Froidefond and S. Ouillon, "Introducing a minicatamaran to perform reflectance measurements above and below the water surface," Opt. Express 13, 926-936 (2005).

11. P. Gege is preparing a manuscript to be called "Analytic model for the direct and diffuse components of downwelling spectral irradiance in water."

12. W. W. Gregg and K. L. Carder, "A simple spectral solar irradiance model for cloudless maritime atmospheres," Limnol. Oceanogr. 35, 1657-1675 (1990).

13. R. Bukata, J. H. Jerome, K. Y. Kondratyev, and D. V. Pozdnyakov, Optical Properties and Remote Sensing of Inland and Coastal Waters (CRC Press, 1995).

14. H. R. Gordon, "Can the Lambert-Beer law be applied to the diffuse attenuation coefficient of ocean water?" Limnol. Oceanogr. 34, 1389-1409 (1989).

15. C. D. Mobley, Light and Water (Academic, 1994).

16. T. I. Quickenden and J. A. Irvin, "The ultraviolet absorption spectrum of liquid water," J. Chem. Phys. 72, 4416-4428 (1980).

17. H. Buiteveld, J. H. M. Hakvoort, and M. Donze, "The optical properties of pure water," Proc. SPIE 2258, 174-183 (1994).

18. K. F. Palmer and D. Williams, "Optical properties of water in the near infrared," J. Opt. Soc. Am. 64, 1107-1110 (1974).

19. A. Morel, "Optical properties of pure water and pure sea water," in Optical Aspects of Oceanography, N. G. Jerlov, E. Steemann Nielsen, eds. (Academic, 1997), pp. 1-24.

20. P. Gege, "Characterization of the phytoplankton in Lake Constance for classification by remote sensing," in Lake Constance-Characterisation of an Ecosystem in Transition, E. Bäuerle, U. Gaedke, eds. (Archiv für Hydriobiologie, 1998), Vol. 53, pp. 179-193.

21. T. Heege, "Flugzeuggestützte Fernerkundung von Wasserinhaltsstoffen am Bodensee," Ph.D. thesis (DLRForschungsbericht, 2000).

22. A. Bricaud, A. Morel, and L. Prieur, "Absorption by dissolved organic matter of the sea (yellow substance) in the UV and visible domains," Limnol. Oceanogr. 26, 43-53 (1981).

23. K. L. Carder, G. R. Harvey, and P. B. Ortner, "Marine humic and fulvic acids: their effects on remote sensing of ocean chlorophyll," Limnol. Oceanogr. 34, 68-81 (1989).

24. J. L. Mueller and R. W. Austin, "Ocean optics protocols for SeaWiFS validation, revision 1," SeaWiFS Technical Report Series, S. B. Hooker, E. R. Firestone, and J. G. Acker, eds., Tech. memo 104566 (NASA, 1995), Vol. 25.

25. G. Zibordi and M. Darecki, "Immersion factors for the RAMSES series of hyper-spectral underwater radiometers," J. Opt. A: Pure Appl. Opt. 8, 252-258 (2006).

26. P. Gege, "The water color simulator WASI: an integrating software tool for analysis and simulation of optical in situ spectra," Comput. Geosci. 30, 523-532 (2004).

27. P. Gege and A. Albert, "A tool for inverse modeling of spectral measurements in deep and shallow waters," in Remote Sensing of Aquatic Coastal Ecosystem Processes: Science and Management Applications, L. L. Richardson and E. F. LeDrew, eds. (Springer, 2006), pp. 81-109.

28. The $\mathrm{R}$ Foundation for Statistical Computing, " $\mathrm{R}$ version 2.11.1," http://www.r-project.org/. 